\title{
Isolated double adrenocorticotropic hormone-secreting pituitary adenomas: A case report and review of the literature
}

\author{
JIUJUN PU, ZHIMING WANG, HUI ZHOU, AILING ZHONG, KAI JIN, \\ LUNLIANG RUAN and GANG YANG
}

Department of Neurosurgery, The First Affiliated Hospital of Chongqing Medical University, Chongqing 410000, P.R. China

Received February 3, 2015; Accepted May 12, 2016

DOI: $10.3892 / \mathrm{ol} .2016 .4673$

\begin{abstract}
Only a few cases of double or multiple pituitary adenomas have previously been reported in the literature; however, isolated double adrenocorticotropic hormone (ACTH)-secreting pituitary adenomas are even more rare. The present study reports a rare case of a 50-year-old female patient who presented with typical clinical features of Cushing's disease and was diagnosed with isolated double ACTH-secreting pituitary adenomas. Endocrinological examination revealed an ACTH-producing pituitary adenoma, and preoperative magnetic resonance imaging (MRI) demonstrated a microadenoma with a lower intensity on the right side of the pituitary gland. The patient underwent endoscopic endonasal transsphenoidal surgery, which revealed another pituitary tumor in the left side of the pituitary gland. The two, clearly separated, pituitary adenomas identified in the same gland were completely resected. Immunohistochemistry and pathology revealed that the clearly separated double pituitary adenomas were positive for ACTH, thyroid-stimulating, growth and prolactin hormones. Postoperatively, the levels of ACTH and cortisol hormone decreased rapidly. The case reported in the present study is considerably rare, due to the presence of a second pituitary adenoma in the same gland, which was not detected by preoperative MRI scan, but was noticed during surgery. Intraoperative evaluation may be important in the identification of double or multiple pituitary adenomas.
\end{abstract}

\section{Introduction}

Isolated double pituitary adenomas are considerably rare. Between 1978 and 2014, only 115 cases of double or multiple pituitary adenomas, including clearly separated and contiguous

Correspondence to: Dr Gang Yang, Department of Neurosurgery, The First Affiliated Hospital of Chongqing Medical University, 1 Yixue Road, Yuzhong, Chongqing 410000, P.R. China

E-mail:yg_angcq@163.com

Key words: isolated double pituitary adenomas, adrenocorticotropic hormone, Cushing's disease, dynamic magnetic resonance imaging, endoscope, case report adenomas, were reported in the literature (1-7). Furthermore, the diagnostic criteria for double or multiple pituitary adenomas vary between pathologists and clinical doctors. Certain cases have been diagnosed on the basis of the expression of $\geq 2$ types of hormones and immunohistochemical staining results by pathologists (5). The combined expression of adrenocorticotropic hormone (ACTH) and prolactin (PRL) is the most common characteristic of double or multiple pituitary adenomas (5); however, immunohistochemical studies on the diagnosis of double or multiple pituitary adenomas have not been fully trusted by clinical doctors when there is cross-reactivity of antisera and an insufficient specimen (8). The doctor making the diagnosis of double or multiple pituitary adenomas should correlate the patient's symptoms with the type of hormone produced by the tumor, since, in the majority of cases, the diagnosis of double or multiple pituitary adenomas is made only on the basis of immunohistochemical staining results. Only a few, clear cases of multiple adenomas have been confirmed by pre- and intraoperative evaluations (6,9-12). If clinicians considered the presence of $\geq 2$ separated tumors in the same pituitary gland or the patient's clinical characteristics pre- and intraoperatively, surgical failure could be prevented to a greater extent.

The present study reports a case of clearly separated adenomas, diagnosed based on preoperative contrast-enhanced magnetic resonance imaging (MRI) findings and surgical exploration. Written informed consent was obtained from the patient.

\section{Case report}

Preoperative findings. A 50-year-old Chinese woman with a history of diabetes mellitus and hypertension presented to The First Affiliated Hospital of Chongqing Medical University (Chongqing, China) with clinical symptoms of Cushing's disease, such as oily skin, moon face, buffalo hump, facial plethora, typical cushingoid central fat distribution and slight purple striae on the abdomen, which had lasted for $>10$ years. Prior to admission on October 24, 2013, endocrine tests revealed that the patient's serum ACTH level was $55.0 \mathrm{pg} / \mathrm{ml}$ and the cortisol (COR) level was $688.9 \mathrm{nmol} / \mathrm{l}$; therefore, the dexamethasone suppression test was performed, and the diurnal variations of ACTH and COR were evaluated. The diurnal variation of both the hormones spontaneously disappeared. The patient's plasma COR and ACTH diurnal variations were as follows: COR, $688.9 \mathrm{nmol} / 1$ and $\mathrm{ACTH}, 55 \mathrm{pg} / \mathrm{ml}$ at 8:00 
a.m.; COR, $500.6 \mathrm{nmol} / \mathrm{l}$ and $\mathrm{ACTH}, 50.45 \mathrm{pg} / \mathrm{ml}$ at 4:00 p.m.; and COR, $614.8 \mathrm{nmol} / \mathrm{l}$ and $\mathrm{ACTH}, 41.23 \mathrm{pg} / \mathrm{ml}$ at 12:00 a.m. The overnight low-dose dexamethasone suppression test (1 mg dexamethasone administered orally at 12:00 a.m.) revealed that low-dose dexamethasone could not suppress the secretion of ACTH and COR, but high-dose dexamethasone $(8 \mathrm{mg}$ orally) suppressed $>50 \%$ of the initial production of COR. The patient was hospitalized due to the highly increased COR and ACTH levels (Table I).

Following admission, the chief complaint of the patient was rapid body-weight gain subsequent to surgery for an ectopic pregnancy in 2000 . The patient's body mass index was $39.56 \mathrm{~kg} / \mathrm{m}^{2}$, and no family history of a pituitary tumor was recorded. Blood biochemical assay results demonstrated blood lipid metabolic disorders: The total cholesterol level was $6.8 \mathrm{mmol} / 1$ (normal range, $2.8-5.2 \mathrm{mmol} / \mathrm{l})$, triglyceride levels were $4.55 \mathrm{mmol} / 1$ (normal range, $0.35-1.7 \mathrm{mmol} / \mathrm{l}$ ), high-density lipoprotein level was $1.08 \mathrm{mmol} / \mathrm{l}$ (normal range, $0.9-1.8 \mathrm{mmol} / \mathrm{l}$ ) and low-density lipoprotein level was $4.05 \mathrm{mmol} / 1$ (normal range, 2.07-3.1 mmol/l). An endocrine examination revealed that all hormone levels, except for ACTH $(53 \mathrm{pg} / \mathrm{ml}$; normal range, 7.20-63.30 pg/ml) and COR (829.07 nmol/1; normal range, 2.74-19.64 nmol/1), were normal (Table II). A preoperative MRI scan detected a 5-mm tumor located on the right side of the pituitary gland (Fig. 1A). Electrocardiography revealed sinus rhythm, and abdominal ultrasonography demonstrated that the adrenal gland was normal. Based on the patient's medical history and radiologic and laboratory findings, an ACTH-secreting pituitary adenoma was diagnosed, and neuroendoscopic surgery was performed. Preoperative preparation included the control of the patient's glycemic and blood pressure and improvement of her nutritional status.

Intraoperative findings. A 5-mm white pituitary microadenoma was visualized on the right side of the pituitary gland. Subsequently, another 3-5-mm white pituitary microadenoma was observed on the left side of the pituitary gland during neuroendoscopic exploration. Based on the findings of the preoperative contrast-enhanced MRI scan (Fig. 1B) and surgical exploration via neuroendoscopy (Fig. 2), the lesions were confirmed as isolated double ACTH-secreting pituitary adenomas, and were completely resected (Fig. 2).

Postoperative findings. The two pituitary tumors obtained during primary surgical resection in October 2013 were analyzed. Pituitary adenoma blocks $(5 \mathrm{~mm})$ were established using formalin-fixed, paraffin-embedded tumor samples. Hematoxylin and eosin staining, which was visualized using an optical microscope (Olympus Corporation, Tokoyo, Japan), revealed that both specimens were adenomas. Subsequently, each pathological section was subjected to immunohistochemical staining with an avidin biotin-peroxidase complex system. Briefly, following deparaffinization and rehydration, epitope retrieval was performed using $10 \mathrm{mmol} / \mathrm{l}$ citrate buffer ( $\mathrm{pH}$ 6.0) for $15 \mathrm{~min}$. The activity of endogenous peroxidases was blocked with $3 \%$ hydrogen peroxide for $10 \mathrm{~min}$ at room temperature. The sections were then incubated with primary rabbit polyclonal ACTH (dilution, 1:200; cat no. ab74976; Abcam), mouse monoclonal growth hormone (GH; dilution, 1:200; cat no. ab7905; Abcam, Cambridge, UK), mouse
Table I. Dexamethasone suppression test.

A, Low-dose overnight dexamethasone suppression test (1 mg orally at 12:00 a.m.)

\begin{tabular}{lcc}
\hline Time of day & ACTH, pg/ml & COR, nmol/1 \\
\hline 8:00 am & 55.00 & 29.06 \\
8:00 am (after 1 day) & 688.9 & 950.8 \\
\hline
\end{tabular}

B, High-dose dexamethasone suppression test (8 mg orally)

\begin{tabular}{lcc}
\hline Time of day & ACTH, pg/ml & COR, nmol/1 \\
\hline 8:00 am & 57.06 & 16.43 \\
8:00 am (after 2 days) & 353.18 & 84.77 \\
\hline
\end{tabular}

$\mathrm{ACTH}$, adrenocorticotropic hormone; COR, cortisol.

monoclonal thyroid-stimulating hormone (TSH; dilution, 1:200; cat no. ab27974; Abcam) and mouse monoclonal PRL (dilution, 1:200; cat no. ab47150; Abcam) antibodies at $4^{\circ} \mathrm{C}$ overnight in a humidified chamber. Negative controls were obtained by the omission of primary antibodies, followed by incubation with polyclonal horseradish peroxidase-conjugated goat antirabbit (1:300; cat. no. ab6112; Abcam) and anti-mouse (1:200; cat. no. ab5879; Abcam) IgG secondary antibodies for $30 \mathrm{~min}$ at room temperature. Slides were stained with 3,3'-diaminobenzidine as the chromogenic substrate for $10 \mathrm{~min}$ in the dark. The slides were then visualized using an optical microscope (Olympus Corporation). Immunohistochemical staining demonstrated that the two adenomas were mainly positive for ACTH and focally positive for TSH, GH and PRL (Fig. 3). The serum hormone levels were measured on postoperative days 1 and 7 . Compared with preoperative levels, the expression of ACTH and COR in the two tumors had markedly decreased following surgery (postoperative day $1,1 \mathrm{pg} / \mathrm{ml} \mathrm{ACTH}$ and $38.47 \mathrm{nmol} / 1$ COR; postoperative day 7, $1 \mathrm{pg} / \mathrm{ml}$ ACTH and $33.01 \mathrm{nmol} / 1$ COR) (Table II). The patient presented with secondary hypoadrenalism and received hydrocortisone replacement treatment.

Three months after surgery, the patient's hormone levels were re-evaluated. Oral glucose tolerance test results revealed impaired glucose tolerance, suggesting that the patient's glycometabolism disorders were recovering slowly following the resection of the pituitary tumors. Dyslipidemia had also improved. The patient had no obvious discomfort.

\section{Discussion}

The incidence rates of clearly separated adenomas are unknown. Magri et al (9) reported that the incidence of double pituitary adenomas in patients who chose not to undergo surgery was $2.6 \%$. Double or multiple pituitary adenomas, and clearly separated adenomas confirmed by preoperative imaging or intraoperative exploration in particular, are considerably rare. Kim et al (10) identified 4 cases $(0.67 \%)$ of multiple adenomas among 600 surgical cases that were suspected to be 
Table II. Preoperative and postoperative hormonal tests.

\begin{tabular}{|c|c|c|c|c|c|}
\hline \multirow[b]{2}{*}{ Hormones } & \multirow[b]{2}{*}{ Reference values } & \multirow[b]{2}{*}{ Preoperative values } & \multicolumn{3}{|c|}{ Postoperative values } \\
\hline & & & Day 1 & Day 7 & Month 3 \\
\hline $\mathrm{GH}, \mathrm{ng} / \mathrm{ml}$ & $0.00-5.00$ & 0.29 & 0.43 & 0.16 & 0.28 \\
\hline $\mathrm{ACTH}, \mathrm{pg} / \mathrm{ml}$ & $7.20-63.30$ & 53 & 1 & 1 & 1 \\
\hline PRL, ng/ml & $2.74-19.64$ & 12.81 & 5.30 & 7.83 & 30.75 \\
\hline $\mathrm{COR}, \mathrm{nmol} / \mathrm{l}$ & $118.64-618.02$ & 829.07 & 38.47 & 33.01 & 64.35 \\
\hline TSH, uIU $/ \mathrm{ml}$ & $0.35-3.50$ & 0.45 & 1.03 & 1.01 & 2.88 \\
\hline $\mathrm{FT} 4, \mathrm{ng} / \mathrm{ml}$ & $0.61-1.12$ & 0.74 & 0.94 & 0.94 & 1.27 \\
\hline $\mathrm{FT}, \mathrm{pg} / \mathrm{ml}$ & $2.19-3.90$ & 2.67 & 2.74 & 3.39 & 3.86 \\
\hline $\mathrm{LH}, \mathrm{mIU} / \mathrm{ml}$ & $1.20-12.86$ & 45.44 & 31.72 & 10.96 & 17.11 \\
\hline $\mathrm{FSH}, \mathrm{mIU} / \mathrm{ml}$ & $1.79-5.12$ & 86.20 & 58.52 & 30.62 & 34.14 \\
\hline Estradiol, ng/ml & 49-291 & 21 & 36 & 30 & 28 \\
\hline Progesterone, $\mathrm{pg} / \mathrm{ml}$ & $0.00-0.78$ & 0.94 & 0.21 & 0.32 & 0.22 \\
\hline
\end{tabular}

GH, growth hormone; ACTH, adrenocorticotropic hormone; PRL, prolactin; COR, cortisol; TSH, thyroid-stimulating hormone; FT, free thyroid hormone; FT4, free thyroxine; LH, luteinizing hormone; FSH, follicle-stimulating hormone.

Table III. Cases of multiple pituitary adenomas.

\begin{tabular}{|c|c|c|c|c|}
\hline \multirow[b]{2}{*}{ Variable } & \multicolumn{2}{|c|}{$\begin{array}{c}\text { Clearly separated } \\
\text { pituitary adenomas }(n=42)^{a}\end{array}$} & \multicolumn{2}{|c|}{$\begin{array}{l}\text { Contiguous } \\
\text { pituitary adenomas }(n=36)^{b}\end{array}$} \\
\hline & $\mathrm{N}$ & $\%$ & $\mathrm{~N}$ & $\%$ \\
\hline \multicolumn{5}{|l|}{ Gender } \\
\hline Female & 29 & 69.0 & 16 & 55.6 \\
\hline Male & 13 & 31.0 & 20 & 44.4 \\
\hline \multicolumn{5}{|l|}{ Preoperative imaging } \\
\hline Magnetic resonance imaging & 22 & 52.4 & 0 & 0.0 \\
\hline Computed tomography & 2 & 4.8 & 0 & 0.0 \\
\hline Intraoperative diagnosis (neurosurgery) & 18 & 42.9 & 0 & 0.0 \\
\hline Postoperative diagnosis (pathology) & 42 & 100.0 & 36 & 100.0 \\
\hline
\end{tabular}

${ }^{\mathrm{a}}$ Median age, 41.5 years; age range, $15-80$ years (1-4,8,10-14,17-25). ${ }^{\mathrm{b}}$ Median age, 45.5 years; age range, 18-76 years $(6-9,11,12,14,26-29)$.

multiple adenomas based on preoperative MRI scan. Ratliff and Oldfield (11) also reported 13 patients (1.82\%) with multiple adenomas that were detected intraoperatively, and 1 patient $(0.15 \%)$ that was diagnosed with multiple adenomas on the basis of preoperative MRI findings.

Following a review of the literature, 115 cases of double or multiple pituitary adenomas were identified (1-7). Based on the classification of double or multiple pituitary adenomas established by Ratliff and Oldfield (11), Andrioli et al (13) and Kontogeorgos et al (14), 37 patients were diagnosed with unclassified multiple pituitary adenomas on autopsy $(15,16)$. The types of adenomas of the remaining 78 patients are listed in Table III. Patients were divided into two groups: Those with clearly separated adenomas and those with a contiguous pituitary tumor diagnosed by pathology.

The youngest reported patient was a 15-year-old girl, whose condition was diagnosed on the basis of MRI findings, according to Goldberg et al (2). The oldest reported patient was 80 years old (8). The majority of patients were aged 41.5-45.5 years. On the basis of preoperative, intraoperative and postoperative evaluations, 42 patients were diagnosed with clearly separated double pituitary adenomas (1-4,8,10-14,17-25), 36 patients with contiguous pituitary adenomas $(6-9,11,12,14,26-29)$, and 3 patients with 3 pituitary adenomas $(11,22,23)$.

The majority of double or multiple pituitary adenomas are mainly detected by one of the following methods: Imaging examination, surgical exploration or autopsy $(9-11,14,16,22,23)$; however, the adenomas nature and type of hormones expressed are confirmed by pathology and immunohistochemistry, respectively (11). At present, in clinical practice, a useful detection method is essential to evaluate the presence of double or multiple pituitary adenomas (9). The coexistence of multiple pituitary tumors often accounts for surgical failure (22). Numerous examination types, including MRI and computed tomography 

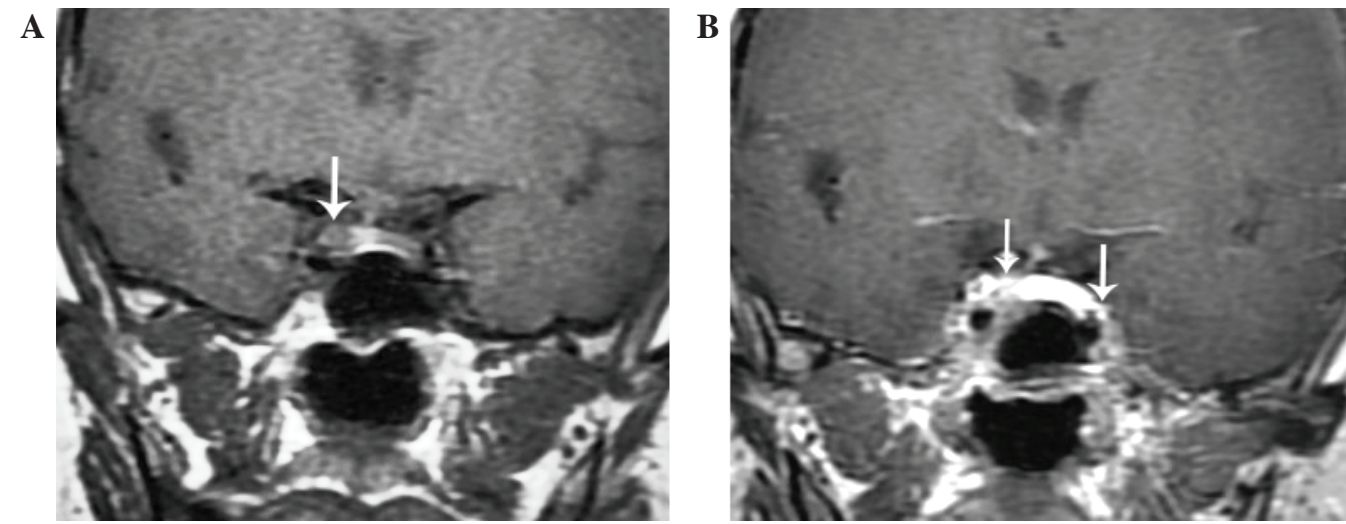

Figure 1. MRI scan of pituitary adenoma. (A) MRI scan of the head identified a 5-mm microadenoma in the right pituitary lobe (arrow); (B) Contrast-enhanced MRI scan of the head revealed two highly suspicious pituitory lesions (arrows). MRI, magnetic resonance imaging.

A

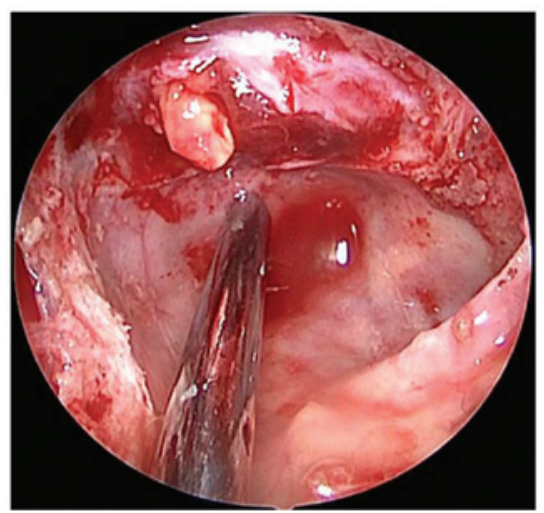

B

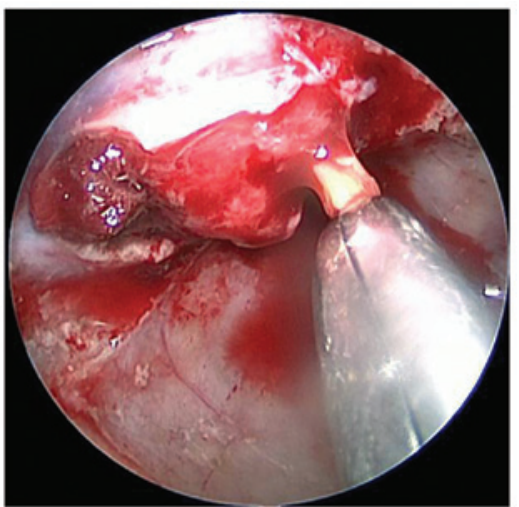

Figure 2. Neuroendoscopic image of double pituitary adenomas. (A) Pituitary adenomas located in the right side of the pituitary gland. (B) Two well demarcated adenomas were separately observed during surgery.

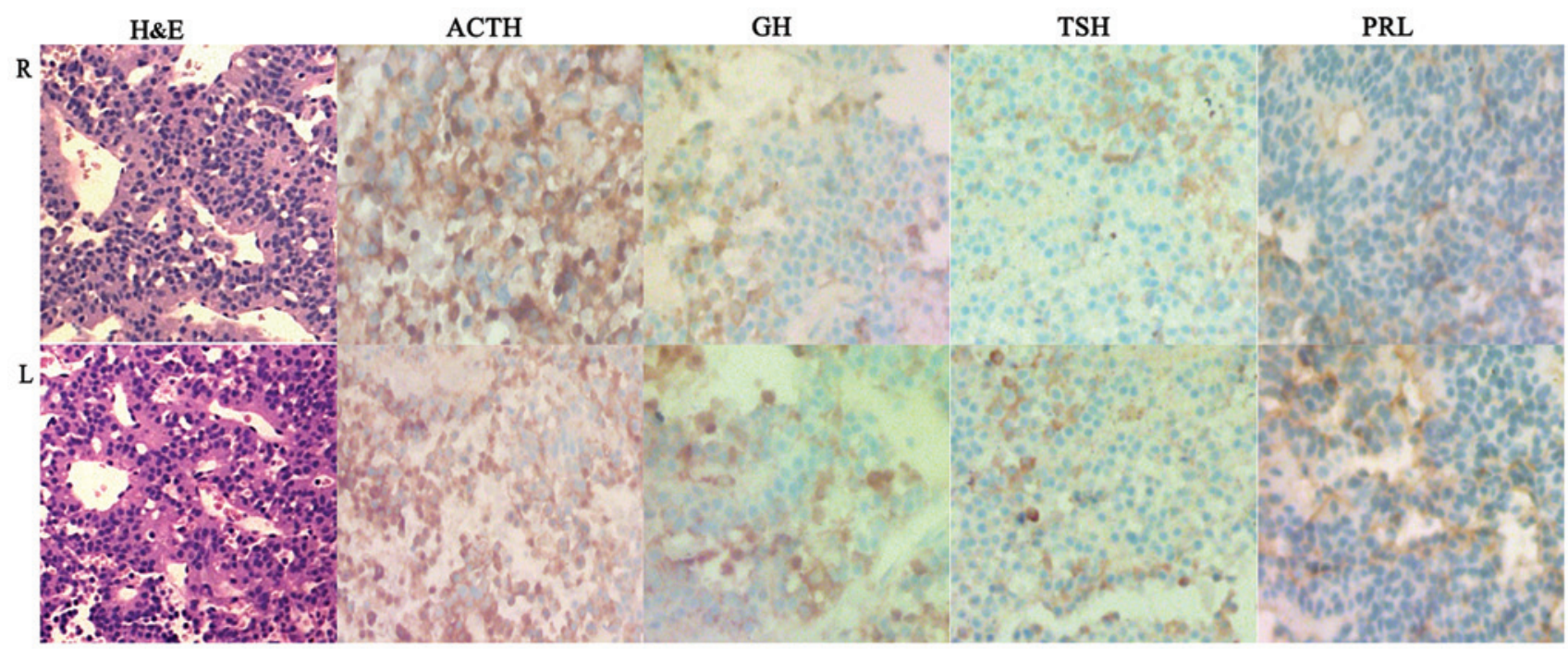

Figure 3. Pathological and immunohistochemical staining findings of the two tumors. Hematoxylin and eosin staining of the two tumors suggested adenomas The cells of both tumors were oval, basophilic cells with clear nucleoli, without signs of focal necrosis, active mitosis or atypical nuclei. Immunohistochemical staining of the two tumors revealed that the cells were mainly positive for adrenocorticotropic hormone and focally positive for thyroid-stimulating, growth and prolactin hormones (magnification, x400). R, right; L, left; H\&E, hematoxylin and eosin; GH, growth hormone; ACTH, adrenocorticotropic hormone; PRL, prolactin; TSH, thyroid-stimulating hormone.

(CT) scans, as well as intraoperative ultrasonography, are able to detect double or multiple pituitary adenomas. MRI was the first reported modality to diagnose multiple pituitary adenomas (22).
Preoperative MRI, particularly thin-slice, dynamic and contrast-enhanced MRI $(2,10,13,17)$, is an effective and sensitive method of determining the presence of multiple 
adenomas $(10-12,14,21,22,29)$. Among 42 patients (Table III), $22(52.4 \%)$ were diagnosed on the basis of preoperative MRI findings, and 2 (4.8\%) on the basis of CT findings (24). The remaining 18 patients were diagnosed during surgery. Preoperative MRI is more important than CT in the diagnosis of multiple adenomas. As indicated in the present case and previous literature reports, contrast-enhanced MRI is able to confirm separated double or multiple pituitary adenomas $(1,3,9,13,17,28)$.

Intraoperative evaluation, including MRI and ultrasonography, as well as surgical exploration, may assist the diagnosis of double or multiple pituitary adenomas $(11,14,18,20,25,30,31)$. A total of 18 cases $(42.9 \%)$ of double pituitary adenomas from the literature were diagnosed during surgery $(11,14,18,20,25,30)$, with the majority of them being clearly visible to the surgeon by the microscopic approach (11). Endoscopic transsphenoidal surgery is used more often than the microscopic approach, particularly in cases of pituitary lesions (32). In the present case, the patient underwent neuroendoscopic transsphenoidal surgery, which allowed for better visualization and enabled the detection of another microadenoma. This prevented the requirement for a second surgery (Fig. 2). Oyama et al (18) reported that 1 patient had to undergo two surgeries to resect two pituitary adenomas; the disease manifestation and hormone levels following the first surgery revealed that the tumors were not in remission, and indicated the presence of another microadenoma. Kontogeorgos et al (14) and McKelvie and McNeill (20) have reported similar cases. Thus, according to Liu et al (33), establishing the difference between a normal pituitary gland and a pituitary adenoma is crucial during surgery.

There are numerous different theories on the pathogenesis of pituitary tumors, including genetic mutations, autocrine/paracrine interactions, altered hypothalamic function, abnormal extrapituitary regulatory mechanisms, epigenetic modification, and microRNAs and large non-coding RNAs such as maternally expressed $3(8,10,34,35)$. Kim et al (10) reported 2 patients with a family history of pituitary adenomas, suggesting that genetic abnormalities may be associated with the pathogenesis of double or multiple pituitary tumors. Kumar and Prusty (4) were the first to report a case of a macroprolactinoma that evolved from two separate microadenomas. It was suggested that a polyclonal origin of the prolactinoma was responsible for the pathogenesis of multiple pituitary adenomas, but there were no definite histopathological results to demonstrate that the original lesion consisted of two prolactinomas. In the present case, the histopathological results demonstrated a pituitary adenoma, and immunohistochemical staining revealed that the tumor cells were positive for ACTH, TSH, GH and PRL (Fig. 3). These findings may explain Kumar and Prusty's conclusions (4).

In summary, progress has been made in determining the roles that tumor suppressors, oncogenes and cycle abnormalities perform during the onset of pituitary adenoma; however, due to the limitations in the use of human pituitary adenoma cell lines and animal models, the acute pathogenesis of multiple pituitary adenomas remains to be fully elucidated $(1,3,4,34,36)$.

\section{References}

1. Kobayashi Y, Takei M, Ohkubo Y, Kakizawa Y, Matoba H, Kumagai M, Takeda T, Suzuki S and Komatsu M: A somatotropin-producing pituitary adenoma with an isolated adrenocorticotropin-producing pituitary adenoma in a female patient with acromegaly, subclinical Cushing's disease and a left adrenal tumor. Endocr J 61: 589-595, 2014.

2. Goldberg AS, Stein R, Merritt NH, Inculet R and Van Uum S: A pediatric patient with Cushing syndrome caused by ectopic ACTH syndrome and concomitant pituitary incidentalomas. J Pediatr Endocrinol Metab 27: 123-128, 2013.

3. Zielinski G, Maksymowicz M, Podgorski J and Olszewski WT: Double, synchronous pituitary adenomas causing acromegaly and Cushing's disease. A case report and review of literature. Endocr Pathol 24: 92-99, 2014.

4. Kumar KV and Prusty P: Resistant prolactinoma: Is it monoclonal or polyclonal? Indian J Endocrinol Metab 17: 2013.

5. Iacovazzo D, Bianchi A, Lugli F, Milardi D, Giampietro A, Lucci-Cordisco E, Doglietto F, Lauriola L and De Marinis L: Double pituitary adenomas. Endocrine 43: 452-457, 2013.

6. Koutourousiou M, Kontogeorgos G, Wesseling P, Grotenhuis AJ and Seretis A: Collision sellar lesions: Experience with eight cases and review of the literature. Pituitary 13: 8-17, 2010.

7. Al Brahim NY, Rambaldini G, Ezzat S and Asa SL: Complex endocrinopathies in MEN-1: Diagnostic dilemmas in endocrine oncology. Endocr Pathol 18: 37-41, 2007.

8. Jastania RA, Alsaad KO, Al-Shraim M, Kovacs K and Asa SL: Double adenomas of the pituitary: Transcription factors Pit-1, T-pit and SF-1 identify cytogenesis and differentiation. Endocr Pathol 16: 187-194, 2005.

9. Magri F, Villa C, Locatelli D, Scagnelli P, Lagonigro MS, Morbini P, Castellano M, Gabellieri E, Rotondi M, Solcia E, et al: Prevalence of double pituitary adenomas in a surgical series: Clinical, histological and genetic features. J Endocrinol Invest 33: 325-331, 2010.

10. Kim K, Yamada S, Usui M and Sano T: Preoperative identification of clearly separated double pituitary adenomas. Clin Endocrinol (Oxf) 61: 26-30, 2004.

11. Ratliff JK and Oldfield EH: Multiple pituitary adenomas in Cushing's disease. J Neurosurg 93: 753-761, 2000.

12. Sano T, Horiguchi H, Xu B, Li C, Hino A, Sakaki M, Kannuki S and Yamada S: Double pituitary adenomas: Six surgical cases. Pituitary 1: 243-250, 1999.

13. Andrioli M, Pecori Giraldi F, Losa M, Terreni M, Invitti C and Cavagnini F: Cushing's disease due to double pituitary ACTH-secreting adenomas: The first case report. Endocr J 57: 833-837, 2010.

14. Kontogeorgos G, Scheithauer BW, Horvath E, Kovacs K, Lloyd RV, Smyth HS and Rologis D: Double adenomas of the pituitary: A clinicopathological study of 11 tumors. Neurosurgery 31: 840-849, 1992.

15. Buurman H and Saeger W: Subclinical adenomas in postmortem pituitaries: Classification and correlations to clinical data. Eur J Endocrinol 154: 753-758, 2006

16. Kontogeorgos G, Kovacs K, Horvath E and Scheithauer BW: Multiple adenomas of the human pituitary. A retrospective autopsy study with clinical implications. J Neurosurg 74: 243-247, 1991.

17. de Oliveira Andrade LJ, Santos França L, Santos França L and Cordeiro de Almeida MA: Double pituitary prolactinoma. J Clin Endocrinol Metab 95: 4848-4849, 2010.

18. Oyama K, Yamada S, Hukuhara N, Hiramatsu R, Taguchi M, Yazawa M, Matsuda A, Ohmura E and Imai Y: FSH-producing macroadenoma associated in a patient with Cushing's disease. Neuro Endocrinol Lett 27: 733-736, 2006.

19. Shimizu C, Koike T and Sawamura Y: Double pituitary adenomas with distinct histological features and immunophenotypes. J Neurol Neurosurg Psychiatry 75: 140, 2004.

20. McKelvie PA and McNeill P: Double pituitary adenomas: A series of three patients. Pathology 34: 57-60, 2002.

21. Tosaka M, Kohga H, Kobayashi S, Zama A, Tamura M, Murakami M and Sasaki T: Double pituitary adenomas detected on preoperative magnetic resonance images. Case illustration. J Neurosurg 92: 361, 2000.

22. Cannavò S, Curtò L, Lania A, Saccomanno K, Salpietro FM and Trimarchi F: Unusual MRI finding of multiple adenomas in the pituitary gland: A case report and review of the literature. Magn Reson Imaging 17: 633-636, 1999. 
23. Pantelia E, Kontogeorgos G, Piaditis G and Rologis D: Triple pituitary adenoma in Cushing's disease: Case report. Acta Neurochir (Wien) 140: 190-193, 1998.

24. Hashimoto N, Handa H, Yamagami T, Kojima M, Aoki M, Okamoto S, Kato Y and Imura H: Acromegaly with multiple secreting pituitary adenomas. Surg Neurol 22: 556-558, 1984.

25. Woosley RE: Multiple secreting microadenomas as a possible cause of selective transsphenoidal adenomectomy failure. Case report. J Neurosurg 58: 267-269, 1983.

26. Rotondo F, Khatun N, Scheithauer BW, Horvath E, Marotta TR, Cusimano $\mathrm{M}$ and Kovacs K: Unusual double pituitary adenoma: A case report. Pathol Int 61: 42-46, 2011.

27. Mohammed S, Cusimano MD, Scheithauer BW, Rotondo F, Horvath E and Kovacs K: O-methylguanine-DNA methyltransferase immunoexpression in a double pituitary adenoma: Case report. Neurosurgery 66: E421-E422, 2010.

28. Coiré CI, Smyth HS, Rosso D, Horvath E and Kovacs K: A double pituitary adenoma presenting as a prolactin-secreting tumor with partial response to medical therapy. Case report. Endocr Pathol 21: 135-138, 2010.

29. Meij BP, Lopes MB, Vance ML, Thorner MO and Laws ER Jr: Double pituitary lesions in three patients with Cushing's disease. Pituitary 3: 159-168, 2000.
30. Tolis G, Bertrand G, Carpenter S and McKenzie JM: Acromegaly and galactorrhea-amenorrhea with two pituitary adenomas secreting growth hormone or prolactin. A case report. Ann Intern Med 89: 345-348, 1978

31. Laws ER: Multiple pituitary adenomas. Journal of neurosurgery 93: 2000.

32. Yadav Y, Sachdev S, Parihar V, Namdev H and Bhatele P: Endoscopic endonasal trans-sphenoid surgery of pituitary adenoma. J Neurosci Rural Pract 3: 328-337, 2012.

33. Liu JF, Ke CS, Chen X, Xu Y, Zhang HQ, Chen J, Gan C, Li CX and Lei T: Identification and management of intra-operative suspicious tissues in 20 transsphenoidal surgery cases. Sichuan Da Xue Xue Bao Yi Xue Ban 44: 441-443, 2013 (In Chinese).

34. Jiang $X$ and Zhang $X$ : The molecular pathogenesis of pituitary adenomas: An update. Endocrinol Metab (Seoul) 28: 245-254, 2013.

35. Melmed S: Pathogenesis of pituitary tumors. Nat Rev Endocrinol 7: 257-266, 2011.

36. Curtò L and Trimarchi F: Comment on: Double, synchronous pituitary adenomas causing acromegaly and Cushing's disease. A case report and review of literature. Endocr Pathol 2013;24:92-99. Endocr Pathol 25: 439-440, 2014. 